\title{
IDENTIDAD ÉTNICA EN INDÍGENAS HUETARES DE QUITIRRISÍ ${ }^{1}$
}

\section{ETHNIC IDENTITY IN HUETARS INDIGENOUS PEOPLE OF QUITIRRISI}

\author{
Marjorie Moreno Salas*
}

RESUMEN

Con una metodología cuantitativa se exploraron algunos elementos que conforman la identidad étnica de los indígenas huetares de Quitirrisí de Mora. Para explorar dicha variable se aplicó un cuestionario a 90 personas de la comunidad. Los resultados indicaron que el grupo mantiene un alto nivel de identidad étnica. Así mismo, algunos factores relevantes para el mantenimiento de la identidad cultural fueron: la tradición de los ancestros, el vivir en la reserva y la sangre indígena. Los datos encontrados en el estudio son relevantes en tanto, abren una perspectiva distinta acerca del "sentimiento de pertenencia" a un grupo étnico.

PALABRAS CLAVES: COSTA RICA * POBLACIÓN INDÍGENA * HUETARES * IDENTIDAD CULTURAL * TRADICIÓN

\section{ABSTRACT}

The article explores some of the elements that are part of ethnic identity of the Huetar indigenous people in Quitirrisi of Mora by means of a quantitative methodology. To investigate such variable, 90 people from the community answered a questionnaire. The results indicated that the group maintains a high level of ethnic identity. Also, some relevant factors for keeping the cultural identity were: their traditions from ancestors, living in the reserve and the indigenous blood. The data revealed some important information since they show a different perspective about the belonging feeling to an ethnic group.

KEY WORDS: COSTA RICA * INDIGENOUS POPULATION * HUETARES * CULTURAL IDENTITY * TRADITION

Este documento presenta resultados que corresponden a uno de los objetivos del trabajo final de graduación de la autora, titulado: "Percepción de discriminación social e identidad étnica en indígenas huetares de Quitirrisí" (2006), el cual formó parte del Proyecto de investigación: Discriminación social: consecuencias psicológicas $y$ estrategias de afrontamiento (723-A6-319) del
Instituto de Investigaciones Psicológicas y recibió financiamiento de la Vicerrectoría de Investigación de la Universidad de Costa Rica.

* Instituto de Investigaciones Psicológicas. Universidad de Costa Rica. maryyuri12@gmail.com 


\section{INTRODUCCIÓN}

En Costa Rica conviven diferentes grupos culturales, entre ellos: afro-costarricenses, blanco-mestizos, migrantes e indígenas. El último grupo resulta de especial interés, debido a sus particularidades $y$ heterogeneidad (en el país existen ocho grupos indígenas), los cuales han sido estudiados desde varias perspectivas, sin embargo, una de las grandes paradojas que enfrentan los habitantes de las comunidades autóctonas se relaciona con mantener sus tradiciones y costumbres, pues actualmente los procesos de aculturación en estos grupos son acelerados.

El presente estudio fue desarrollado con el grupo Huetar, específicamente la Reserva de Quitirrisí, del total de grupos indígenas costarricenses, esta comunidad es la que se ubica más cerca del Valle Central. Dicha comunidad se encuentra en el cantón de Mora, entre los límites de Guayabo y Tabarcia (Ornat, 2002).

Ahora bien, de acuerdo con los datos del Censo del 2000, la mayoría de sus pobladores laboran en el sector terciario de la economía (área de servicios) y gran parte de esta población se desplaza diariamente a San José (capital de Costa Rica). Sin duda, este patrón productivo constituye un punto de interés pues incrementa las posibilidades de contacto inter-grupal.

Para el 2004 la población total de la reserva alcanzó 751 personas (datos proporcionados por el Área de Salud de Mora). La extensión de la misma es de 2660.03 hectáreas (datos proporcionados por José Manuel Paniagua funcionario de la Comisión Nacional de Asuntos Indígenas (CONAI), comunicación telefónica, mayo 2005).

Respecto de los procesos identitarios y de aculturación, en el pasado Bozzoli $(1969,1973)$, citada por Quesada (1996), excluyó a los huetares de los grupos indígenas costarricenses (comunidades de Zapatón y Quitirrisí), pues en estudios etnográficos reportó no haber encontrado manifestaciones de identidad étnica; paradójicamente, años después sí los incorporó, lo cual podría significar de acuerdo con Quesada (1996), que este grupo ha retornado a sus raíces, es decir, se ha generado a lo interno de la comunidad un proceso particular de fortaleci- miento del sentido de pertenencia a un grupo étnico.

Por otro lado, en cuanto a las prácticas culturales, Quesada (1996) expresó que aún conservan -en medio de los diferentes encuentros culturales-, trabajos con material textil, obtención de tintes, elaboración de utensilios caseros, comidas y bebidas.

Sin embargo, generalmente la identidad étnica se asocia de manera directa con preservar las costumbres, el idioma, el traje tradicional, las comidas, la música y los ritos funerarios (la mayoría no están presentes en el grupo Huetar). Pero la información recopilada hasta el momento sobre el grupo en estudio, señala que este no conserva el vestido tradicional, el idioma, la religión y otros aspectos señalados.

Ahora bien, en primera instancia al efectuar una integración de los factores señalados previamente es posible afirmar que los huetares mantienen prácticas bi-culturales. Esto desde la perspectiva teórica de Berry (1997), quien plantea que mientras en el proceso de aculturación se muestran transformaciones culturales que surgen del encuentro o contacto entre dos grupos culturales, en el nivel psicológico se señalan los cambios que experimentan las personas en aspectos subjetivos como la identidad étnica, sus actitudes, sus valores y sus conductas.

Es decir, cuando se plantea la bi-culturalidad se hace referencia a Berry (1997) y los planteamientos orientados a indicar que los sujetos pueden pertenecer a diferentes grupos culturales $y$ mantener identificaciones afectivas con más de un grupo social.

De manera que, cuando se señala que los indígenas son un grupo bi-cultural se hace referencia a que manifiestan presencia de elementos de ambas culturas: la indígena y la blanca mestiza. Se considera que lo anterior es potenciado por la fuerte relación laboral con San José. Otras actividades que denotan el proceso de aculturación son: celebrar los quince años de las adolescentes, la celebración del rito funerario católico $y$ en el aspecto religioso, pues la mayoría de las personas se denominó a sí misma como católica o cristiana, (Ornat, 2002).

Por último, al considerar las condiciones de vida de los indígenas quitirriseños, y 
la información documentada en los trabajos de Bozzoli y los aportes de Quesada, surge el cuestionamiento: actualmente ¿cuáles elementos conforman la identidad étnica de los quitirriseños?

\section{ANTECEDENTES}

Algunos de los antecedentes del presente estudio, corresponden a los trabajos realizados por Phinney; para profundizar en el tema se recomienda consultar: Phinney (1989), Phinney \& Tarver (1989), Phinney (1990), Phinney (1992), Phinney, Chavira \& Tate (1992).

Por otra parte, el antecedente directo del estudio realizado lo constituye el trabajo de Ornat (2002). La investigación se centró en el proceso de etno-génesis que se gestaba en la comunidad. Ornat, citando a Wallace (1956) propone la etno-génesis como "los esfuerzos deliberados, organizados y conscientes de los miembros de una sociedad para la construcción de una cultura más sofisticada” (p. 278). La autora realizó un recorrido histórico desde la era precolombina, hasta que el territorio fue declarado reserva. El objetivo general fue: analizar el proceso - conjunto y divergente- de etno-génesis que se está produciendo en la comunidad de Quitirrisí desde su conformación como reserva/territorio indígena.

Los datos de Ornat respecto de la identidad étnica señalaron que los quitirriseños conservan el medio ambiente, algunas palabras de su idioma, utensilios de cocina, conocimientos en cuanto a flora y fauna, comidas tradicionales, artesanías, leyendas y creencias. De la misma forma, la autora indicó que los pobladores originarios de la comunidad de Quitirrisí se han apropiado de elementos particulares de la cultura mestiza como: la estructura de las viviendas, los electrodomésticos (televisor y lavadora), el vestido, los productos de limpieza, el calendario católico, la celebración de los quince años, el rito funerario y las leyendas no indígenas.

Respecto de aspectos subjetivos, Ornat (2002) asegura que en la comunidad existe orgullo por la diferencia cultural. Pero de la misma forma, algunos adolescentes se niegan a asumirse como indígenas. Mientras que, per- sonas adultas manifiestan melancolía por el idioma y traje típico. Finalmente, como base de la identidad étnica, la autora aseguró que esta población considera el fenotipo, pues lo asocian con sus características físicas y principalmente la sangre indígena. Es decir, este grupo en cuanto a la identidad étnica le atribuyen gran importancia al aspecto físico y el conocimiento de la ascendencia indígena.

Los resultados de Ornat brindan una perspectiva diferente respecto del grupo indígena, pues se refieren a estilos de vida particulares, e integrando la descripción anterior, este grupo se puede caracterizar nuevamente como "bicultural", debido a que por un lado conservan aspectos de sus antepasados y por el otro se han "apropiado" de prácticas del grupo blancomestizo.

Cabe recalcar que el presente estudio fue realizado en el 2006, varios años después del efectuado por Ornat (2002) y como es de esperarse las condiciones en cualquier contexto social varían conforme transcurren los años.

Para dar respuesta a lo planteado previamente, es relevante esclarecer algunos conceptos. La identidad étnica, en este estudio se define como el sentido de pertenencia a un grupo étnico y a la parte de los procesos emocionales, de acción y pensamiento que se derivan del reconocimiento de la propia etnicidad, Smith (2002a), citando a Phinney \& Rosenthal (1992).

Sin embargo, la identidad étnica se ha conceptualizado como un aspecto duradero $y$ fundamental del yo, que incluye un sentimiento de conexión a un grupo social o étnico, las actitudes y sentimientos asociados con el grupo (Yen \& Hwang, 2000). Los mismos autores citando a Phinney (1995) sostienen que la identidad étnica consiste en factores tales como el ser marcado, un sentido de pertenencia, la preferencia por el grupo étnico, el interés, el conocimiento y la participación en actividades asociadas con el grupo.

El grupo étnico es conceptualizado como un grupo humano que se distingue por tener características culturales comunes entre sí, pero diferentes a las de otros grupos humanos, lo anterior de acuerdo con Smith (2002a) citando a Duncan \& Powel (1988). 
La misma autora citando a Phinney (1990) delimita la etnicidad como el hecho concreto de nacer dentro de un grupo étnico particular, sin excluir la posibilidad de pertenecer a dos o más grupos étnicos.

La ley indígena 6172, en el artículo primero, define indígenas como "aquellas personas que constituyen grupos étnicos descendientes directos de las civilizaciones precolombinas $y$ que conservan su propia identidad". Sin embargo, respecto a la etnicidad, se priorizó la dimensión psicológica y subjetiva de los procesos de pertenencia, dejando de lado factores externos para centrarse en el discurso de las personas $y$ sus expresiones acerca de sentirse indígenas 0 no y las motivaciones que externaron para ello.

En el presente estudio se consideró indígenas a las personas que aseguraron haber nacido en Quitirrisí y que durante la recolección de datos a la pregunta ¿usted es indígena Huetar? contestaron afirmativamente.

Para enmarcar el fenómeno de la identidad étnica, se recurrió a los planteamientos de Turner (1984) sobre la identidad grupal, quien plantea que la hipótesis básica respecto a la identidad del grupo es que el comportamiento grupal depende del efecto cognoscitivo de la categorización social, la auto-definición y autopercepción. Es decir, los conceptos anteriores: identidad étnica, grupo étnico, persona indígena $y$ etnicidad tienen sentido en la medida en que confluyen en el sujeto con el sentido de pertenencia al grupo social.

Mientras que, otro elemento relevante fue el auto-concepto, en este caso considerado el sistema de conceptos disponible a la persona para procurar definirse a sí misma. Este sería una estructura cognoscitiva hipotética que media entre las situaciones sociales y el comportamiento. En el auto-concepto se pueden identificar dos componentes principales, la identidad social y la personal. La primera está vinculada con las auto-descripciones relacionadas con el número de miembros del grupo de pertenencia, formal e informal, entre ellos, el sexo, la nacionalidad, la ocupación, la religión y la identidad étnica.

Según Turner (1984), la identidad social produce comportamientos y actitudes de grupo $y$ es el mecanismo cognoscitivo que permite el comportamiento grupal. El proceso de la categorización se refiere a la percepción de estímulos en términos de atributos comunes para definirse $y$ el perfeccionamiento consecuente de su similitud con miembros del mismo grupo, así como desigualdad con los miembros de clases diferentes.

Los individuos se perciben principalmente por lo que se refiere al número de miembros de grupo, ellos/as se asignan los atributos estereotipados asociados al mismo.

Algunos de los rasgos distintivos del comportamiento de grupo, de acuerdo con Turner (1984) serían: similitud percibida entre los miembros; atracción mutua, estima, cooperación y altruismo; uniformidades compartidas en actitudes y comportamientos, contagio emocional y empatía.

Como se argumentó, las teorías de la identidad y categorización social, se han centrado en el funcionamiento del grupo, y en la medida en que los individuos se comprometen con este. Queda claro que se trata de un aspecto cognoscitivo que permite al sujeto integrarse al grupo y calificarlo de determinada forma.

De igual importancia resultan los planteamientos teóricos sobre la identidad social —de acuerdo con van Knippenberg, (1984) —el cual la define como la parte del auto-concepto de una persona que se deriva de su conocimiento del número de miembros de su grupo social (o grupos), junto con el valor y la importancia emocional atribuida a estos. La identidad social define el lugar particular que el individuo ocupa en la sociedad. El aspecto central de la teoría de la identidad social es que los individuos se esfuerzan por una identidad social positiva; van Knippenberg (1984), citando a Tajfel (1978).

Siguiendo el planteamiento anterior caben de nuevo las preguntas ¿Qué aspectos articulan la identidad étnica de los indígenas huetares? ¿Qué permite a este grupo étnico mantener sus tradiciones e identidad? y ¿Cuáles son los mecanismos que utilizan para continuar valorando positivamente su grupo?, pues queda claro que el grupo mestizo es el dominante en la sociedad costarricense.

Junto con lo anterior, la situación de las personas pertenecientes a grupos minoritarios 
se agrava en una sociedad con fuertes ideas importadas, que fomentan el consumo, la individualidad $y$ tratan de eliminar las diferencias, esperando que las personas se comporten de la misma forma, consuman los mismos productos, hablen el mismo idioma y lleven a cabo las mismas prácticas culturales. Así mismo, no es "ningún secreto" que los grupos indígenas costarricenses no poseen el valor social que deberían, pese a la diversidad y riqueza cultural que representan para Costa Rica, cuentan con un estatus social bajo y es factible considerarlos "grupos minoritarios".

Por consiguiente, el proceso de aculturación es avanzado, pero aún está presente la parte del sí mismo que liga a la persona con su grupo, raíces, historia y demás aspectos emocionales que permiten a los indígenas de Quitirrisí denominarse como tales.

A continuación, se presenta información relacionada con la metodología que se empleó para la recolección de información.

\section{MÉTODO}

Con el propósito de establecer el nivel de identidad étnica y determinar los principales aspectos que según los indígenas de esta región conforman su sentido de pertenencia, se recurrió a una metodología cuantitativa. El diseño del estudio fue transversal y de tipo descriptivocorrelacional.

Durante la fase de recolección de datos, se elaboró y validó un cuestionario, se contactaron habitantes de la comunidad, $y$ aplicaron 90 instrumentos a personas mayores de edad, que fueron ubicadas en sus viviendas.

Para responder a la pregunta de investigación se utilizaron varias mediciones. Al inicio del cuestionario, se solicitaron los datos sociodemográficos. En la segunda parte se presentaron una serie de afirmaciones con el propósito de determinar cuáles elementos culturales eran considerados clave por los indígenas respecto de su identidad, (Ej. Yo me siento un indígena Huetar de Quitirrisí porque: Tengo sangre indigena, hablo el idioma indígena de mi grupo). Las categorías de respuesta fueron $0=$ No, $1=$ Sí.
Seguidamente, se preguntó: ¿De 1 a 10, qué tan indígena se siente usted?, con el propósito de contar con una segunda medición de la identidad étnica, además, se solicitó justificar la respuesta.

Por último, las personas de la comunidad completaron la Escala de Identidad Étnica Multigrupo (EIEM) en la versión adaptada por Smith (2002b) al contexto costarricense, la cual consta de nueve ítems, y posee una consistencia interna indicada por coeficientes Alfa de Cronbach superiores a 0,80 . Uno de los ítems fue "Estoy consciente de mis raíces étnicas y lo que eso significa para mí". Las categorías de respuestas fueron: $1=$ Prácticamente nunca, $2=$ Muy pocas veces, $3=$ Varias veces y $4=$ Casi siempre.

Para el análisis de los resultados se empleó el Paquete Estadístico para Ciencias Sociales (SPSS), versión 11.0.

\section{RESULTADOS}

A continuación se presentan los datos sociodemográficos de la población, las características psico-métricas del instrumento y los resultados obtenidos.

La primera interrogante del cuestionario correspondió a la pregunta, ¿A cuál grupo étnico pertenece usted? Pese a que el total de las personas encuestadas vivían en la reserva $y$ se declararon indígenas, de los 90 participantes (53 mujeres y 37 hombres), solamente 82 contestaron al "grupo Huetar", las restantes ocho manifestaron respuestas como: "grupo Quitirrisî”, o "de aquí de Quitirrisî”. Lo cual sugiere un desconocimiento de su origen cultural. El promedio de edad de los/as participantes fue 35,27 años y la moda 19 años.

La comunidad está dividida en barrios o caseríos, la mayoría de las personas habitaban: Centro de Quitirrisí ( $n=29)$, Alto de Quitirrisí $(n=11)$ y Barrio San Juan $(n=9)$.

La distribución geográfica es un aspecto cualitativo substancial, pues la comunidad cuenta con una estructura particular, ya que el territorio está dividido por una autopista. Se considera que lo anterior contribuye de manera parcial con la dificultad para ubicar un centro 
de reunión, sitios públicos, o espacios sociales que construyan "comunidad".

Sintetizando otros datos, predomina la población del centro de Quitirrisí, de religión católica, con una pareja estable y que poseen primaria completa.
La tabla 1 resume los resultados psicométricos de la escala de identidad étnica multigrupo. Hubo 84 personas que completaron el total de reactivos y el coeficiente de Alpha de Cronbach alcanzó 0,89.

TABLA 1

MEDIA, DESVIACIÓN ESTÁNDAR Y CORRELACIÓN DE LOS ÍTEMS DE LA ESCALA DE IDENTIDAD ÉTNICA

\begin{tabular}{|c|c|c|c|}
\hline ÍTEMS & M & SD & $r_{i t}$ \\
\hline 1. Estoy feliz de ser un indígena Huetar & 3,51 & 0,94 & 0,60 \\
\hline 2. Me siento fuertemente ligado (a) a mi grupo Huetar & 2,68 & 1,25 & 0,72 \\
\hline 3. Me siento muy comprometido (a) con mi grupo Huetar & 2,53 & 1,25 & 0,76 \\
\hline 4. Me siento muy bien con mi tradición cultural & 3,20 & 1,05 & 0,71 \\
\hline 5. Estoy muy orgulloso(a) de mi grupo Huetar & 3,02 & 1,18 & 0,76 \\
\hline 6. Estoy consciente de mis "raíces huetares" y de lo que estas significan para mí & 3,19 & 1,03 & 0,71 \\
\hline $\begin{array}{l}\text { 7. Asisto a grupos y organizaciones que están compuestas, en su mayoría, por huetares } \\
\text { 8. Participo activamente de las tradiciones huetares, como por ejemplo }\end{array}$ & 2,36 & 1,14 & 0,54 \\
\hline $\begin{array}{l}\text { de su comida, artesanías, etc. } \\
\text { 9.Asisto a organizaciones y participo en actividades en donde se promueven }\end{array}$ & 2,69 & 1,16 & 0,63 \\
\hline las tradiciones huetares & 2,50 & 1,18 & 0,48 \\
\hline Número de ítems & & 9 & \\
\hline Número de casos & & 84 & \\
\hline Coeficiente Alpha de Cronbach & & 0,89 & \\
\hline
\end{tabular}

Nota. Las categorías de respuesta fueron de 1 a 4, a mayor puntaje mayor identidad étnica.

De la escala de identidad étnica, el ítem con mayor correlación fue: "Estoy muy orgulloso/a de mi grupo Huetar" con un puntaje de 0,76, seguidamente se ubicó el reactivo "Me siento muy comprometido con mi grupo Huetar" con un puntaje de 0,75.

En cuanto al análisis de los resultados, sobre elementos culturales importantes para las personas de Quitirrisí, se realizó una prueba $\mathrm{t}$ para determinar si se presentaban diferencias por género, edad o zona geográfica, los resultados no arrojaron diferencias significativas, (ts $<1,69$, ns) lo que indica que los análisis que se presentan a continuación aplican de igual forma para toda la muestra.

La tabla 2 presenta el porcentaje de respuesta (se reportan solo los datos correspondientes a la categoría Sí) para cada elemento propuesto, es decir se sumó la cantidad de personas que marcó la opción 1.
TABLA 2

\section{ELEMENTOS CULTURALES DE INTERÉS PARA LOS/AS QUITIRRISEÑOS}

\section{ELEMENTOS CULTURALES}

PORCENTAJE

1. Vivo en Quitirrisí

$96,7 \%$

2. Tengo sangre indígena

$93,3 \%$

3. Mis padres son indígenas

$87,8 \%$

4. Consumo comidas tradicionales

$84,4 \%$

5. Estoy convencido de mi descendencia indígena

$82,0 \%$

6. Tengo rasgos indígenas $\quad 75,6 \%$

7. Simplemente me siento unido/a a mi grupo $57,8 \%$

8. Sé mucho de las tradiciones y costumbres huetares

9. Conozco la historia de mi grupo indígena

10. Me curo con medicina tradicional $43,3 \%$

11. Conozco la música de mi grupo $\quad 42,2 \%$

12. Practico tradiciones indígenas de mi grupo $28,9 \%$

13. Conozco la religión de mi grupo indígena $\quad 23,3 \%$

14. Vivo en una casa tradicional de los huetares $13,3 \%$

15. Hablo el idioma indígena de mi grupo $\quad 5,6 \%$

16. Utilizo el traje tradicional $\quad 4,4 \%$


A los informantes se les presentaron una serie de elementos culturales $y$ anotaron si los consideraban relevantes o no. A continuación se revisan los porcentajes de acuerdo para cada aspecto. Se consideran de especial relevancia los componentes que cuentan con un acuerdo mayor al 75\%, los cuales fueron: "tengo sangre indígena, mis padres son indígenas, consumo comidas tradicionales, estoy convencido de mi descendencia indígena, $y$ tengo rasgos indígenas".

Lo anterior permite establecer como ejes principales la ascendencia (que el padre, madre o abuelo/a sean indígenas) y lo ligado a esta como la apariencia física, el hecho de formar parte de una reserva indígena y como un tercer aspecto la tradición cultural, dentro de lo que están las comidas tradicionales.

Por otro lado, los aspectos asociados con elementos tradicionalmente considerados "identitarios" se ubican en puntajes menores al 50\%, tales como: "conozco la música de mi grupo, práctico tradiciones indígenas de mi grupo, conozco la religión de mi grupo indígena, vivo en una casa tradicional de los huetares, hablo el idioma indígena de mi grupo, y utilizo el traje tradicional". Lo anterior muestra una tendencia clara a valorar como aspectos centrales elementos históricos y concretos, en tanto se asocian con la reserva indígena a la cual pertenecen.

Seguidamente, se consultó por medio de una pregunta abierta por otros elementos que formaran parte de la identidad étnica (las personas debían completar la frase: otra cosa que me hace indígenas es...), las respuestas facilitadas se especifican en la tabla 3. El procedimiento realizado con la información fue agrupar las respuestas abiertas en categorías.

TABLA 3

OTRAS COSAS QUE LOS/AS HACEN INDÍGENAS

\begin{tabular}{|c|c|c|}
\hline RESPUESTAS & $\%$ & CATEGORÍAS \\
\hline $\begin{array}{l}\text { "Vivo en mi reserva" } \\
\text { "Por el lugar" } \\
\text { "Por nacer aquí" } \\
\text { "El pueblo" }\end{array}$ & $\begin{array}{l}8,6 \% \\
5,8 \% \\
2,9 \% \\
2,9 \%\end{array}$ & Nacer y vivir en la reserva indígena \\
\hline $\begin{array}{l}\text { "Mis padres son indígenas" } \\
\text { "De parte de mi mamá tengo sangre indígena" } \\
\text { "Mi descendencia" } \\
\text { "Por mi familia" }\end{array}$ & $\begin{array}{l}5,7 \% \\
2,9 \% \\
2,9 \% \\
2,9 \%\end{array}$ & Los antepasados \\
\hline $\begin{array}{l}\text { "Mi tradición" } \\
\text { "Hacer artesanía" } \\
\text { "Mis comidas tradicionales" } \\
\text { "Trabajar en cosas como canastos, sombreros" } \\
\text { "Mis costumbres" } \\
\text { "Costumbres, actividades comunales" } \\
\text { "Me gustan las comidas y fiestas de } \\
\text { la comunidad" }\end{array}$ & $\begin{array}{c}11,4 \% \\
11,4 \% \\
5,8 \% \\
5,7 \% \\
2,9 \% \\
2,9 \% \\
2,9 \%\end{array}$ & $\begin{array}{c}\text { Tradiciones del grupo } \\
\text { Huetar }\end{array}$ \\
\hline $\begin{array}{l}\text { "Por los derechos indígenas" } \\
\text { "Ser como ellos y no ser explotados por otros" } \\
\text { "Rasgos físicos" } \\
\text { "No avergonzarme de ello" } \\
\text { "Su humildad y responsabilidad para sobrevivir" } \\
\text { "Aceptar lo que soy y lo que ser indígena me da" }\end{array}$ & $\begin{array}{l}2,9 \% \\
2,9 \% \\
2,9 \% \\
2,9 \% \\
2,9 \% \\
2,9 \%\end{array}$ & Otras categorías \\
\hline Número de participantes & 35 & \\
\hline
\end{tabular}


Los mayores puntajes corresponden a: "mi tradición, hacer artesanías", respuestas que se ubicaron en una categoría llamada: "tradiciones del grupo Huetar", en orden de importancia se ubicaron "vivo en mi reserva y mis padres son indígenas", respuestas que fueron agrupadas en otras categorías, algunas de ellas: "nacer $y$ vivir en la reserva, $y$ los antepasados”.

Cabe mencionar el alto porcentaje $(11,4 \%)$ que obtuvo "hacer artesanía" y es posible asociarlo con la considerable cantidad de personas de la comunidad que elaboran materiales como canastos y bolsos, algunos de estos se comercializan en puestos ubicados en la carretera al cantón de Puriscal. Esta tradición es trascendente, pues es una práctica indígena autóctona.

Respecto de la escala de identidad étnica, el promedio del grupo fue 2,84 (las categorías de respuesta eran de 1 a 4 , a mayor puntaje mayor identidad étnica). Posteriormente, se realizó un análisis de correlación de Pearson entre la escala de identidad étnica multi-grupo y la pregunta ¿de 1 a 10 qué tan indígena se siente usted?

El resultado fue un coeficiente de correlación alto y significativo, lo cual indica que ambas son aspectos de un mismo constructo $(\mathrm{r}=0,61, \mathrm{p}<0,01)$.

Igualmente, respecto de la pregunta ¿De 1 a 10 qué tan indígena se siente usted? la moda fue $10, y$ la media 7,24. Específicamente, un $35,3 \%$ marcó el puntaje 10 , un $28,2 \%$ de las personas se ubicó en el 5 ; los demás puntajes son diversos, lo cual demuestra una fuerte vinculación con el grupo, pues adicionalmente solamente un $8,2 \%$ de las personas se sitúo en puntajes iguales o menores a 4 .

Seguidamente, se les solicitó justificar el dato, es decir, las personas indicaron a que se debió el puntaje elegido en la pregunta de 1 a 10.

La tabla 4 contiene las respuestas de 73 personas, que contestaron puntajes superiores a cinco, con esta información se construyeron categorías: la primera corresponde a "Nacer y vivir en la reserva indígena" dentro de la cual se ubicaron respuestas como: "nací, crecí y vivo en la reserva, porque vivo en la reserva" otra de las categorías corresponde a la "sangre indígena" representada por: "por los rasgos, tengo sangre indígena" seguidamente se ubicó la "tradición cultural" con respuestas como: "por ser indígena puro, artesano, por mi apariencia".

TABLA 4

ARGUMENTOS PARA LAS RESPUESTAS DE ALTOS PUNTAJES EN LA PREGUNTA DE 1 A 10

\begin{tabular}{lcc}
\hline RESPUESTAS & $\%$ & CATEGORÍAS \\
\hline "Nací y crecí y vivo en la reserva" & $4,1 \%$ & $2,7 \%$ \\
"Porque vivimos en la reserva y tenemos derechos" & $2,7 \%$ & Nacer y vivir en la reserva \\
"Nacer y crecer en la comunidad" & Indígena \\
"Porque vivo en la reserva" & $1,4 \%$ & "La comunidad" \\
"Soy de aquí" & $1,4 \%$ & Sangre indígena \\
"Por ser nacido aquí" & $1,4 \%$ & $1,4 \%$ \\
"Soy nativa y me gusta el lugar donde nací" & $1,4 \%$ & $1,4 \%$ \\
"Por ser de la reserva" & & \\
"Por el lugar del nombre" & $5,5 \%$ & \\
"Por los rasgos" & $5,5 \%$ & $1,4 \%$ \\
"Llevo sangre indígena" & $1,4 \%$ & \\
"Por mi apariencia" & & \\
\hline "Por las indígenas que lo otro" & $6,8 \%$ &
\end{tabular}


Continuaciòn...

"Por comidas indígenas"

"Por ser indígena puro, artesano"

"Por todos los rasgos culturales de mis antepasados"

Tradición cultural

"Mis antepasados eran indígenas"

$1,4 \%$

"Mis padres y abuelos son indígenas"

$4,1 \%$

$4,1 \%$

$2,7 \%$

$1,4 \%$

$1,4 \%$

$1,4 \%$

$1.4 \%$

$1,4 \%$

$1,4 \%$

"Mis papas nacieron en Quitirrisî"

"Por ser indígena pura"

$2,7 \%$

$1,4 \%$

$1,4 \%$

Otras

"Porque me gusta mucho lo indígena"

73

Por otro lado, quienes anotaron bajos puntajes en la pregunta de 1 a 10 (inferiores a 5), justificaron sus respuestas fundamentalmente con dos aspectos: la pérdida de la tradición cultural y la pérdida de la sangre indígena; es decir, el hecho de que el padre o la madre no sean de la reserva es un elemento que las personas mencionaron como justificación de sus bajos puntajes.

Cabe mencionar que estas variables pueden ser comprendidas como una estra- tegia en sí misma, pues se incorpora la raíz histórica y se brinda poco valor a aspectos reconocidos tradicionalmente como propios de los grupos étnicos, es decir, el vestido tradicional, el idioma y las costumbres. Es así como, la ausencia de aspectos tradicionales no impide que este grupo continúe sintiéndose indígena $y$ valorando sobre todo la descendencia, el legado de los antepasados y la conexión con la tierra.

TABLA 5

ARGUMENTOS PARA LOS BAJOS PUNTAJES EN LA PREGUNTA DE 1 A 10

\begin{tabular}{|c|c|c|}
\hline RESPUESTAS & PORCENTAJE & CATEGORÍAS \\
\hline "Porque casi no paso aquí y no converso con nadie" & $2,7 \%$ & \multirow{8}{*}{$\begin{array}{l}\text { Pérdida de } \\
\text { tradiciones culturales }\end{array}$} \\
\hline "Porque las cosas han cambiado mucho" & $2,7 \%$ & \\
\hline "No practico las tradiciones y costumbres" & $2,7 \%$ & \\
\hline "Perdemos un poco las costumbres" & $2,7 \%$ & \\
\hline "Va pasando" & $1,4 \%$ & \\
\hline "Casi no se practican tradiciones y costumbres" & $1,4 \%$ & \\
\hline "No practico la cultura y no me relaciono con gente de aquî" & $1,4 \%$ & \\
\hline "La comunidad no práctica las tradiciones" & $1,4 \%$ & \\
\hline "Porque mi papá no es indígena" & $5,5 \%$ & \multirow{3}{*}{$\begin{array}{c}\text { Pérdida de la } \\
\text { sangre indígena }\end{array}$} \\
\hline "Por ser cruzado" & $1,4 \%$ & \\
\hline "Solo mi mama es indígena" & $1,4 \%$ & \\
\hline "Porque no son como los de Guatuso" & $1,4 \%$ & \multirow{2}{*}{$\begin{array}{c}\text { Comparación con } \\
\text { otros grupos indígenas }\end{array}$} \\
\hline "Porque no tengo el apoyo" & $1,4 \%$ & \\
\hline Número de participantes & 13 & \\
\hline
\end{tabular}


Los datos anteriores coinciden con los encontrados por Ornat (2002), pues pese a la aculturación y la ausencia de elementos considerados tradicionalmente determinantes, existe una alta identidad étnica en esta comunidad $(M=2,84)$, lo anterior posee importantes repercusiones a los niveles teórico y práctico, pues aportan datos significativos acerca de la predominancia de los factores psicológicos, es decir, puede que un grupo experimente una enorme cantidad de transformaciones, pero mientras exista la conciencia de ser "un grupo", de pertenencia $y$ de tener particularidades, existe la identificación con el mismo pese a los diversos cambios $y$ transformaciones sociales.

\section{DISCUSIONES Y CONCLUSIÓN}

La identidad étnica, comúnmente se ha conceptualizado como un aspecto asociado con elementos observables, en este caso se procuró revisar aspectos psicológicos ligados a sentirse parte de un grupo étnico. Llama la atención que pese a la relativa inexistencia de elementos culturales como la vestimenta, la religión y el idioma; los y las indígenas de Quitirrisí reportaron un alto nivel de identidad étnica, y por tanto, sienten orgullo de su grupo. Lo anterior señala la pertinencia de cuestionar los procesos de transformación social que incluyen elementos subjetivos como la historia del grupo, y la sangre indígena.

La reflexión debe estar orientada a generar discusión acerca de la cuestionada pérdida de identidad étnica de estos grupos, pues al nivel cultural las transformaciones son observables, y cada vez más deben ser consideradas aspectos que generan y mantienen identidad. En este grupo predominan elementos históricos y psicológicos particulares que casualmente tienden a contraponerse con los aspectos señalados como "pérdida de identidad", tales como que los padres no sean de la comunidad, o que no posean sangre indígena.

Este estudio aporta información que apoya elementos subjetivos de la identidad étnica, y por tanto, sería necesario incorporar en los diferentes estudios de índole cultural variables relacionadas con la auto-adscripción étnica y los componentes culturales que han experimentado transformaciones sociales.

Por otra parte, los indígenas de Quitirrisí continúan sintiéndose orgullosos del grupo étnico al que pertenecen, lo cual es muy relevante, pues como se ha revisado a lo largo del presente documento, han atravesado diferentes momentos históricos. En un inicio, según Bozzoli no mostraron identidad étnica, pero posteriormente sí fueron considerados un grupo indígena costarricense.

En el 2002 Ornat, exploró el proceso de "etnogénesis" es decir, el proceso de formación de la etnia, momento en el cuál llegó a resultados similares a los presentes, es decir: "en la comunidad se siente orgullo por el grupo étnico".

Los datos parecen indicar que este nivel de identidad se ha mantenido alto, al considerar importantes la descendencia indígena, la historia de los/as abuelos/as y el vivir en la reserva. Igualmente, predomina la dimensión psicológica, el recuerdo, el anhelo, el orgullo y la historia del grupo.

Además, pareciera que las pocas tradiciones que se conservan, reciben considerable atención y son fomentadas en diferentes espacios, tal es el caso de la elaboración de canastos $y$ textiles.

Paralelo a los procesos afectivos, se hipotetiza que existen una serie de procesos cognitivos que generan identidad, es decir, pareciera que no es fundamental la práctica de las tradiciones o la creencia en las mismas, el hecho de que el padre/madre hayan nacido en la reserva es suficiente para sentirse parte del grupo. Otro aspecto que se repite en diferentes ocasiones es el estar convencido/a y el creer que se es indígena $y$ no tener intenciones de cambiar.

Además, la tradición y el sentido de pertenencia están funcionando como un sello distintivo y que consolida el nexo grupal.

Es relevante que este grupo carece de elementos visibles, pero por el contrario cuenta con aspectos subjetivos.

Finalmente, cómo se mide que alguien es indígena o no, en este caso se consideró la auto-adscripción étnica, es decir, el hecho de que una persona de la comunidad indicara ser indígena. 
Ahora bien, en futuras investigaciones es importante explorar ¿qué sucede con los pobladores que se han desplazado a territorios no indígenas?, ¿continúan sintiéndose parte del grupo cultural en que nacieron?, ¿qué significa para ellos/as ser indígenas?, esto es relevante, en tanto la sociedad costarricense está mostrando cada vez más transformaciones culturales, económicas y sociales, por ello, se considera fundamental impulsar procesos que permitan que los grupos culturales del país dialoguen acerca de sí mismos, ¿qué desean para sus familias y próximas generaciones, en cuanto a la conservación de tradiciones, las prácticas y la cultura en general?

Además, se considera necesario indagar estos aspectos con grupos que viven lejos de las cabeceras de provincias, para examinar ¿qué sucede cuando se tiene a disposición elementos como el vestido, el idioma y las comidas tradicionales?

Por último, es sumamente alentador (aún en esta época) contar con grupos de personas que mantengan sus tradiciones, costumbres e identidad étnica, sin duda esto enriquece nuestro país, la cultura y ello significa una oportunidad para potenciar ciudadanos educados, sensibles, pero especialmente respetuosos de las diferentes prácticas culturales.

\section{BIBLIOGRAFÍA}

Berry, J. "Immigration, acculturation, and adaptation”. Applied Psychology. 1997.

Deschamps, J. C. "The social psychology of intergroup relations and categorical differentiation". Tajfel, H. (Ed). The social dimension. Cambridge. Cambridge University Press, 1984: 541- 558.

Ley indígena 6172. Asamblea Legislativa de la República de Costa Rica. 16 de noviembre de 1977.

Ornat, R. El proceso de etnogénesis en Quitirrisí: Identidad y poder. [Tesis de maestría en Antropología]. Universidad de Costa Rica. 2002.
Phinney, J. "The multi-group ethnic identity measure: A new scale for use with adolescents and young adults from diverse groups". Journal of Adolescent Research 7. 1992: 156-176.

Phinney, J, Chavira, V., \& Tate, J. "The effect of ethnic threat on ethnic self-concept and own-group ratings". The Journal of Social Psychology 133. 1992: 469-478.

Phinney, J. "Ethnic identity in adolescents and adults: Review or research". Psychological Bulletin 108. 1990: 499-514.

Phinney, J. "Stages of ethnic identity development in minoritary group Adolescents". Journal of Early Adolescence 9. 1989: 34-49.

Phinney, J. \& Tarver, S. "Ethnic identity search and commitment in black and white eighth graders". Journal or Early Adolescence 8. 1989: 265- 227.

Quesada, M. Los huetares: Historia, lengua etnográfica y tradición oral. Cartago: Editorial Tecnológica de Costa Rica, 1996.

Smith, V. "La psicología social de las relaciones intergrupales: modelos e hipótesis". Revista Actualidades en Psicología 20. 2006: 45-71.

Smith, V. "Discriminación percibida y autoestima en jóvenes de grupos étnicos minoritarios $y$ mayoritarios en Costa Rica". Revista Interamericana de Psicología 39. 2005: 1-14.

Smith, V. Acculturation and psychology adaptation. CT: Greenwood Press, 2003.

Smith, V. "Aportes a la comprensión de la identidad étnica en niños, niñas $y$ adolescentes de grupos étnicos minoritarios". Revista de Ciencias Sociales 2. 2002a: 71-82. 
Smith, V. "La escala de identidad étnica multigrupo (EIEM) en el contexto costarricense: aportes al estudio de la identidad étnica $y$ sus componentes en jóvenes de diversos grupos étnicos". Actualidades en Psicología 18. 2002b: 83-113.

Turner, J. C. "Social identification and psychological group formation".

Tajfel, H. (Ed). The social dimension. Cambridge. Cambridge University Press, 1984: 518-536. van Knippenberg. "Intergroup differences in group perceptions". Tajfel, H. (Ed). The social dimension. Cambridge. Cambridge University Press, 1984: 561-577.

Yeh, C. J. \& Hwang, M. Y. "Interdependence in ethnic identity and self: implications for theory and practice". Journal of Counseling \& Development 78. 2000: 420-430. 